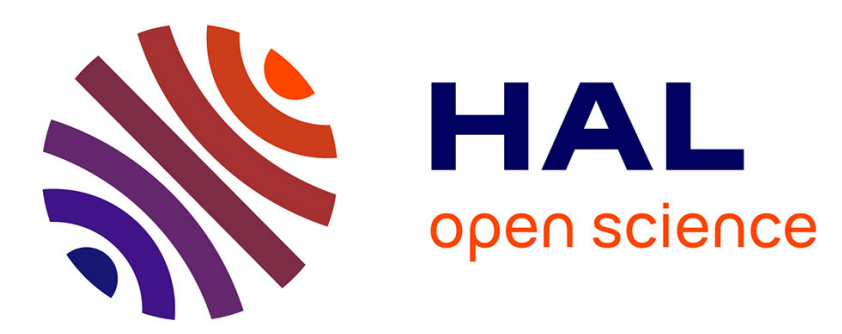

\title{
Distributed Heuristic Algorithms for RAT Selection in Wireless Heterogeneous Networks
}

\author{
Farah Moety, Marc Ibrahim, Samer Lahoud, Kinda Khawam
}

\section{To cite this version:}

Farah Moety, Marc Ibrahim, Samer Lahoud, Kinda Khawam. Distributed Heuristic Algorithms for RAT Selection in Wireless Heterogeneous Networks. IEEE Wireless Communications and Networking Conference (WCNC), Apr 2012, Paris, France. hal-00702544

\section{HAL Id: hal-00702544 https://hal.inria.fr/hal-00702544}

Submitted on 30 May 2012

HAL is a multi-disciplinary open access archive for the deposit and dissemination of scientific research documents, whether they are published or not. The documents may come from teaching and research institutions in France or abroad, or from public or private research centers.
L'archive ouverte pluridisciplinaire HAL, est destinée au dépôt et à la diffusion de documents scientifiques de niveau recherche, publiés ou non, émanant des établissements d'enseignement et de recherche français ou étrangers, des laboratoires publics ou privés. 


\title{
Distributed Heuristic Algorithms for RAT Selection in Wireless Heterogeneous Networks
}

\author{
Farah Moety* $^{* \dagger}$, Marc Ibrahim ${ }^{\dagger}$, Samer Lahoud ${ }^{\ddagger}$, Kinda Khawam ${ }^{\S}$ \\ *Université Libanaise - Ecole Doctorale de Sciences et Technologie, Lebanon \\ ${ }^{\dagger}$ Ecole Supérieure des Ingenieurs de Beyrouth, Lebanon \\ $\ddagger$ University of Rennes I - IRISA, France \\ $\S$ Université de Versailles - PRISM Laboratory, France
}

\begin{abstract}
In wireless heterogeneous networks, one of the most challenging problems is Radio Access Technology (RAT) selection that must be designed to avoid resource wastage. In this paper we adopt a hybrid model for RAT selection where the system allocates the downlink traffic between two different technologies in order to enhance global performance. We study the case of an integrated hybrid Wireless Local Area Network environment where the challenge we face is the high computational complexity necessary to obtain the global optimal solution. Therefore, we propose four distributed heuristic algorithms for RAT selection, where two of them are based on the distance between the user and the access points (APs), namely, distance based and probabilistic distance based algorithms. While the two others schemes are based on the peak rate that each user receives from these APs (peak rate based and probabilistic peak rate based algorithms). Results show that the proposed algorithms give efficient results compared to the optimal one depending on the spatial users distribution. Moreover these algorithms have a low computational complexity which makes them more advantageous compared to the optimal scheme in presence of a large number of users.
\end{abstract}

\section{INTRODUCTION}

The next wireless generation is characterized by the coexistence of a variety of wireless access technologies such as Universal Mobile Telecommunications System (UMTS), Wireless Local Area Network (WLAN), Worldwide Interoperability for Microwave Access (WiMAX), Long Term Evolution (LTE), etc. These different RATs can be integrated together to complement each other in terms of coverage, bandwidth, services, and mobility support, thus providing benefits for both operator and users. The user connected to the "best network" benefits from a good quality of service (QoS) at low cost. The operator in turn can better exploit its radio resources, satisfy more customers and offer new services.

In this heterogeneous context, a new challenging problem of RAT selection arises. RAT selection is the functionality devoted to decide to which RAT a given user will be associated, where the challenge is to optimize for example the QoS, the throughput, or the cost of the network. In [1], three categories of RAT selection schemes are considered based on the parameters taken into account in decision making, namely, service-based, load balancing-based, and interferencebased algorithms. A service-based RAT selection scheme uses a direct mapping between services and RATs [2]. A load balancing strategy distributes the load among different RATs as evenly as possible [3]; specifically, the selected RAT will be the one having the lowest load. An interference-based strategy takes transmitted power level, propagation conditions, interference, and specific network characteristics into consideration [4]. Different approaches for RAT selection were studied such as individual, global and hybrid approaches. In the individual approach, each user selfishly strives to improve its own performance; authors in [5] studied this approach in an integrated WLAN and UMTS hybrid cell where users decide to join one of the two RATs so that their average service time is optimized. In [6], authors studied the global approach where the system load balances the downlink traffic of every user between two RATs WiMAX and WiFi in a way to privilege the overall system performances. The hybrid approach [7] combines the two previous ones and benefits from feedback in order to enhance local decision.

In this paper, we adopt the global approach where the system allocates the downlink traffic between two different technologies. Based on our work in [6], we formulate this approach as an optimization problem that consists in minimizing the total delay defined as the sum of all individual delays. Then we study the case where we have two types of WiFi [8] technologies $(802.11 \mathrm{~b}$ and $802.11 \mathrm{~g}$ ) as if we had two different RATs. The challenge we face here is the high computational complexity to obtain the optimal solution for a large number of users. Therefore, as a contribution to our previous work where we just focused on the formulation of the RAT selection problem and gave a solution based on game theory, we propose here four distributed heuristic algorithms for RAT selection. Then we evaluate by simulation the performance of these algorithms with respect to the optimal one. Three different scenarios are considered regarding the spatial users distribution for this evaluation.

The rest of the paper is organized as follows. The system model is described in Section II. The studied $802.11 \mathrm{~b}$ and $802.11 \mathrm{~g}$ scenario is described in Section III. The proposed heuristic algorithms are presented in Section IV. Simulation results are given in Section V. Conclusions are given in Section VI.

\section{System Model ON THE DOWNLINK AND ASSUMPTIONS}

We consider a geographic region that is entirely covered by a primary RAT and partly covered with several hotspots 
of a secondary RAT as shown in Figure 1. Only mobile users present in secondary RAT hotspots benefit from load balancing their traffic between the two available RATs. The other mobile users profit only from the primary RAT coverage. Also we assume that we are in a static state of the network and users are stationary. In other words, we take a snapshot of a dynamic system and optimize its current state; hence, we consider that the calculation is performed for each user arrival/departure. Furthermore, we assume that the system is in a saturation state which means that we treat a worst case scenario where every user has persistent traffic.

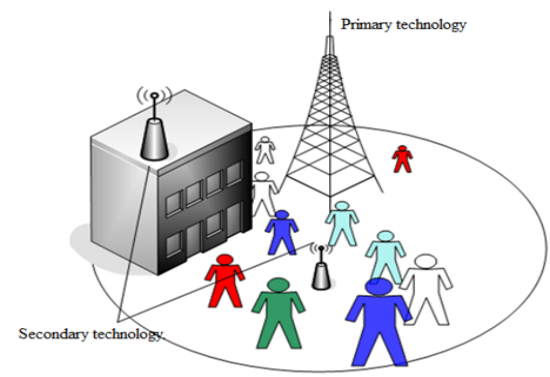

Figure 1. Hybrid wireless access environment

\section{A. Network Structure}

The index $x$ is used throughout the paper to designate a given cell, $x=0$ for the primary RAT and $x=1, \ldots, H$ for the $H$ secondary RAT hotspots. We denote by $n_{x}$ the number of users able to connect to cell $x$. Since all users can connect to the primary RAT, $n_{0}$ is the total number of mobile users and $\bar{n}=n_{0}-\sum_{x=1}^{H} n_{x}$ is the number of users that are only serviced by the primary RAT. Thus, only $n=\sum_{x=1}^{H} n_{x}$ users are engaged in the RAT selection; such users will be indexed by $k=1, \ldots, n$ and will be coined hybrid users. We term by $h(k)$ the secondary RAT hotspot of user $k$. Note that even if we have several hotspots $h(k)$, a user $k$ can be connected only to one $h(k)$.

\section{B. Traffic Model}

We denote by $\theta_{k}$ the instantaneous fraction of time (or the percentage of traffic) during which user $k$ is assigned to its secondary RAT hotspot. Hence, $1-\theta_{k}$ is the remaining fraction of time spent by user $k$ in the primary RAT. We assume the existence of a central entity responsible of routing the downlink traffic of each user either to the primary or secondary RAT depending on the user association.

\section{Cost Function}

We denote by $T_{k, x}$, the cost function of user $k$ when sending all its traffic in $\operatorname{RAT} x . T_{k, x}$ will be a function of the percentage of traffic of all other users $\theta_{-k}$ in the cell. Thus the cost in the primary RAT is:

$$
T_{k, 0}=f_{0}\left(1-\theta_{-k}\right)
$$

and in the secondary RAT is:

$$
T_{k, h(k)}=f_{h(k)}\left(\theta_{-k}\right)
$$

Therefore, the cost function of a hybrid user $k$, denoted by $C_{k}\left(\theta_{k}\right)$ is given by:

$$
C_{k}\left(\theta_{k}\right)=T_{k, 0} \cdot\left(1-\theta_{k}\right)+T_{k, h(k)} \cdot \theta_{k}
$$

\section{Non Linear Problem}

In the global approach, the system load balances the downlink traffic of each hybrid user among RATs in order to optimize the total cost. Thus, this approach can be formulated as an optimization problem $(\mathcal{P})$ that consists in minimizing the total network cost. This cost, denoted by $C_{t}$, is defined as the sum of all individual user costs and is given by: $C_{t}\left(\theta_{k}, k \in N\right)=\sum_{k=1}^{n} C_{k}\left(\theta_{k}\right)$, where $N$ denotes the set of hybrid users. Consequently, the optimization problem $(\mathcal{P})$ is given by:

$$
\begin{gathered}
(\mathcal{P}): \text { Minimize } C_{t}\left(\theta_{k}, k \in N\right)=\sum_{k=1}^{n} C_{k}\left(\theta_{k}\right) \\
\text { Subject to: } 0 \leq \theta_{k} \leq 1, k=1, . ., n
\end{gathered}
$$

\section{Heterogeneous WiFi Scenario}

The model we developed above is applicable to different types of technologies. As the 802.11 WLANs are being widely deployed, we study the case where we have two kinds of WiFi technologies as if we had two different RATs, considering the $802.11 \mathrm{~g}$ technology as the primary RAT and the $802.11 \mathrm{~b}$ technology as the secondary one.

\section{A. Radio Conditions}

In each cell, the peak rate of each user depends on its radio conditions. Let $\chi_{k, x}$ denote the instantaneous peak rate of user $k$ in cell $x$. Each hybrid user perceives two peak rates $\chi_{k, 0}$ and $\chi_{k, h(k)}$ depending on the chosen RAT.

\section{B. Data Rate Model in WiFi}

As we consider only the downlink traffic and neglect the 802.11 waiting times (i.e DIFS, SIFS), the CSMA/CA access scheme leads to a fair rate sharing of the channel among users. Therefore, all users have the exact same throughput. The throughput in question is less than the minimum peak rate that a single user can perceive when alone in the cell [9]. Therefore, the data rate of user $k$ in $802.11 \mathrm{~g}$ is:

$$
R_{k, 0}=1 / \sum_{i=1}^{n_{0}} \frac{\mathbb{1}_{\{\text {user i admitted in } 802.11 \mathrm{~g}\}}}{\chi_{i, 0}}
$$

where

$\mathbb{1}_{\{\text {user i admitted in } 802.11 \mathrm{~g}\}}= \begin{cases}1 & \text { if user i admitted in } 802.11 \mathrm{~g}, \\ 0 & \text { if user i admitted in } 802.11 \mathrm{~b} .\end{cases}$ and the data rate of user $k$ in $802.11 \mathrm{~b}$ hotspot is:

$$
R_{k, h(k)}=1 / \sum_{\substack{i \\ h(i)=h(k)}}^{n} \frac{\mathbb{1}_{\{\text {user i admitted in 802.11b hotspot } \mathrm{h}(\mathrm{k})\}}}{\chi_{i, h(i)}}
$$




\section{Cost Function}

We consider that $T_{k, x}$, the cost function of user $k$ in cell $x$ adopted in the theoretical fair rate model, is the amount of time necessary to send a data unit in cell $x$. In other words, the cost function is the inverse image of the throughput. In 802.11g, we deduce from Equation 4 the following:

$$
T_{k, 0}=\mathbb{E}\left[1 / R_{0}\right]=\frac{1}{\chi_{k, 0}}+\sum_{i=1, i \neq k}^{n_{0}} \frac{1}{\chi_{i, 0}}+\sum_{i=1, i \neq k}^{n} \frac{1-\theta_{i}}{\chi_{i, 0}}
$$

The same applies in 802.11b hotspot $h(k)$ using Equation 5:

$$
T_{k, h(k)}=\mathbb{E}\left[1 / R_{k, h(k)}\right]=\frac{1}{\chi_{k, h(k)}}+\sum_{\substack{i=1, i \neq k \\ h(i)=h(k)}}^{n} \frac{\theta_{i}}{\chi_{i, h(i)}}
$$

Therefore, the cost function of a hybrid user $k$ given in Equation 3 will be its expected time necessary to send a unit of data in the hybrid environment.

\section{Optimal Solution}

In the previous section, the global performance is formulated as the non linear optimization problem $(\mathcal{P})$. In [6], the authors demonstrated that the global optimal solution for this problem is always reached when $\theta_{k}=1$ or $\theta_{k}=0$ for each user $k, k=1, \ldots, n$. Therefore each user is assigned either to the $802.11 \mathrm{~g}$ or to the $802.11 \mathrm{~b}$ hotspot.

The time complexity for computing the optimal solution is $\mathcal{O}\left(2^{n}\right)$, where $n$ is the number of users. Moreover we need a central entity that has an overall knowledge of the network. Therefore, we are going to introduce distributed heuristic algorithms for RAT selection in the next section.

\section{Distributed HeuristiC Algorithms}

Due to the high computational complexity of the global optimal solution, we propose four distributed heuristic algorithms for RAT selection starting with a simple algorithm based on the distance between the user and each of the primary and secondary access points (APs). In the second one, in order to give the possibility to distribute users between the two technologies, we add uncertainty to the selection so that the probability to connect to one technology is inversely proportional to the distance between the user and the corresponding AP. The third algorithm is based on the peak rate that each user receives from the two technologies. Finally in the last algorithm, to not connect all users to the broader technology, we combine peak rate and probability in a manner the probability to connect to one technology is proportional to the peak rate received by the user from this technology.

\section{A. Distance Based Selection}

In this algorithm we take into consideration the distance between the user and each of the two APs where the user connects to the nearest AP. In fact, this distance reflects the power of the received signal at the user side. Algorithm 1 describes the different steps of the distance based selection algorithm.
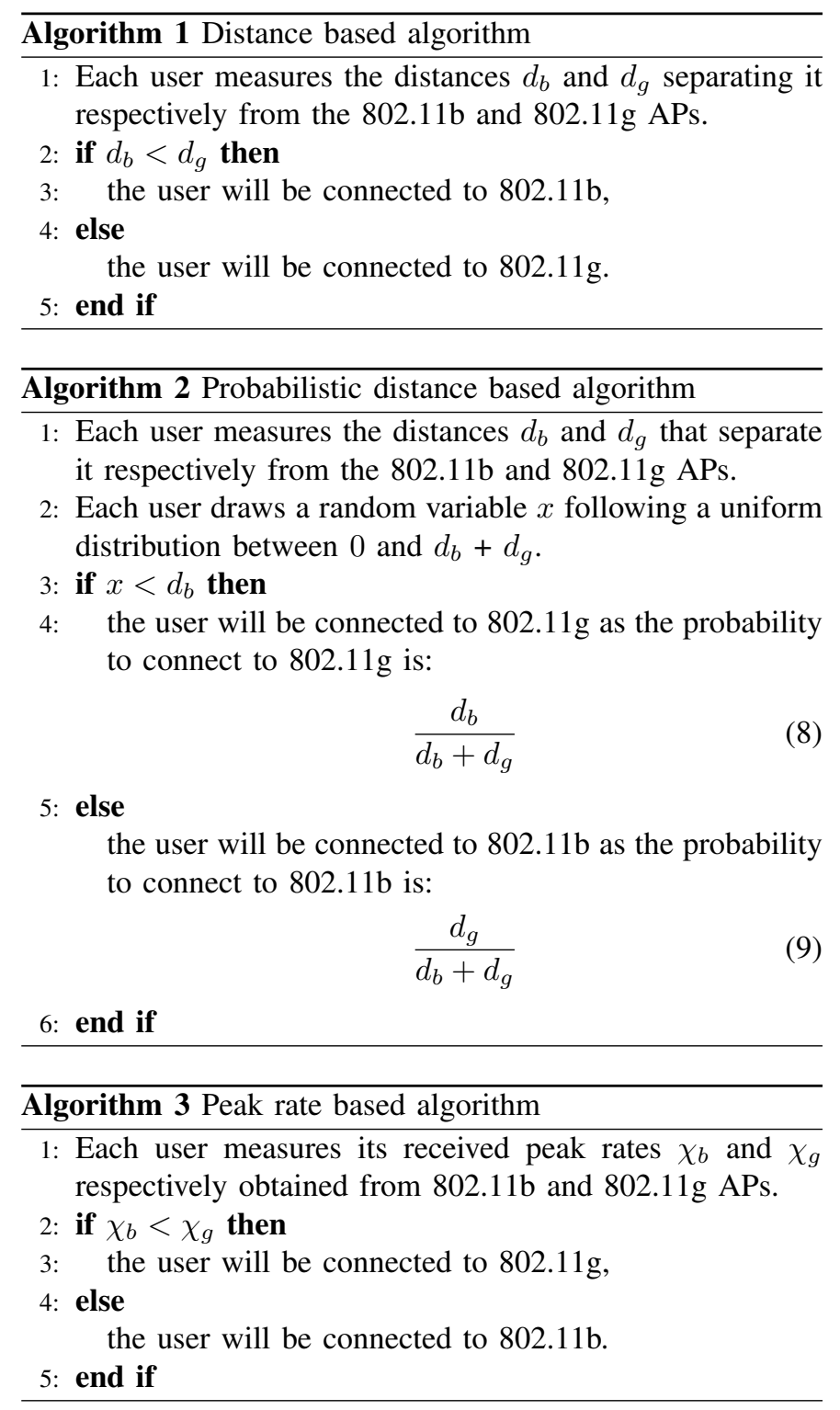

\section{B. Probabilistic Distance Based Selection}

As mentioned earlier, in this algorithm we combine the distance to a probability function in a way that the probability to connect to a given technology is inversely proportional to the distance between the user and the corresponding AP. Therefore, the closer the user is to the AP, the higher its probability to be connected to this AP. The different steps considered in this algorithm are shown in Algorithm 2.

\section{Peak Rate Based Selection}

This algorithm is based on the peak rate each user receives from the two technologies in such a way the user connects to the RAT where it gets the maximum peak rate. The different steps of this algorithm is described in Algorithm 3.

\section{Probabilistic Peak Rate Based Selection}

In this algorithm, we combine the peak rate with a probability function. We obtain that the probability to connect to 


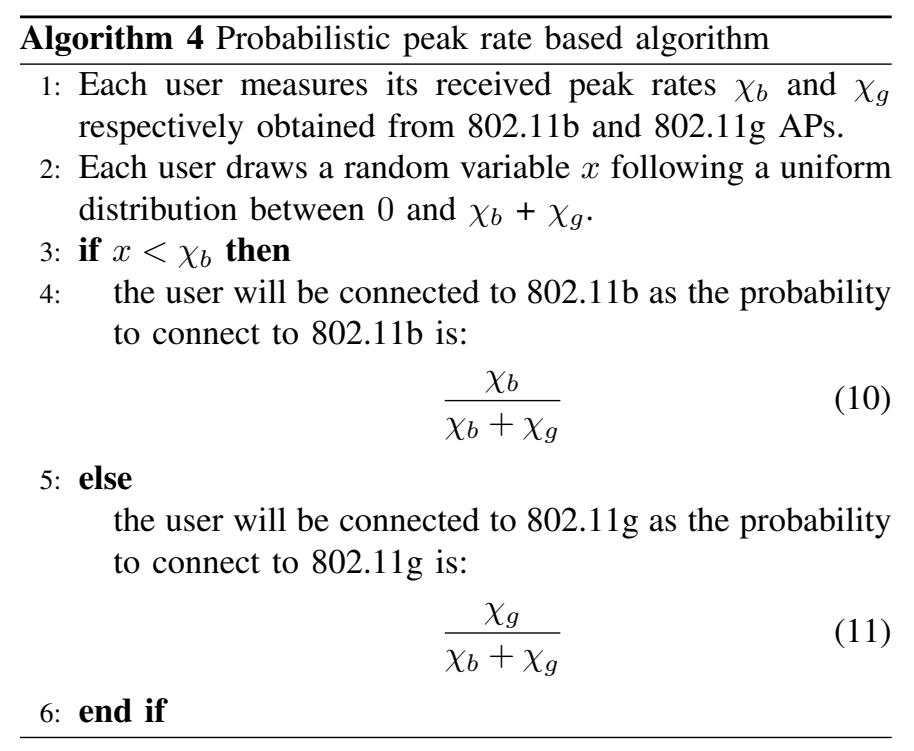

a given technology is proportional to the peak rate received by the user from the corresponding AP. Thus, the higher the peak rate received from the AP, the higher will be the user probability to be connected to this AP. The different steps considered in this algorithm are shown in Algorithm 4.

In order to evaluate the efficiency of these algorithms we will compare by simulation the global optimal solution to the results obtained by these heuristic algorithms in the next section.

\section{Simulation Results}

Simulations in this work are done using NS2 Network Simulator. We consider a geographic region that is entirely covered by $802.11 \mathrm{~g}$ and partly covered by a $802.11 \mathrm{~b}$ hotspot. There are 12 hybrid users generated randomly following a uniform distribution. These users are downloading a Pareto traffic over User Datagram Protocol (UDP) with a Burst Time of $400 \mathrm{~ms}$, an Idle Time of $100 \mathrm{~ms}$, a source Data Rate of $20 \mathrm{Mb} / \mathrm{s}$ (which leads to saturation state of the network according to the assumption presented in section II) and an average Packet Size of 1000 Bytes.

We consider three different scenarios regarding the spatial users distribution with respect to the WiFi APs:

- Scenario I: $50 \%$ of users are closer to the $802.11 \mathrm{~g}$ AP.

- Scenario II: $75 \%$ of users are closer to the 802.11 g AP.

- Scenario III: $25 \%$ of users are closer to the $802.11 \mathrm{~g}$ AP.

For each scenario, five users association schemes are simulated. The first scheme implements the optimal global solution while the four others correspond to the heuristic approaches. More precisely, for each scenario, we calculate the total cost defined as the sum of all individual costs for the optimal solution and compare it with the costs obtained by the proposed heuristic algorithms. For simplicity, we denote by $O$ the simulated global network cost for the optimal solution, by $D$ the distance based solution, by $P D$ the probabilistic distance based solution, by $R$ the peak rate based solution, and by $P R$

\begin{tabular}{|r|r|r|r|r|}
\hline Ratio 1 & Ratio 2 & Ratio 3 & Ratio 4 & Ratio 5 \\
\hline $\mathrm{D} / \mathrm{O}$ & $\mathrm{PD} / \mathrm{O}$ & $\mathrm{PD} / \mathrm{D}$ & $\mathrm{R} / \mathrm{O}$ & $\mathrm{R} / \mathrm{D}$ \\
\hline Ratio 6 & Ratio 7 & Ratio 8 & Ratio 9 & Ratio 10 \\
\hline $\mathrm{R} / \mathrm{PD}$ & $\mathrm{PR} / \mathrm{O}$ & $\mathrm{PR} / \mathrm{D}$ & $\mathrm{PR} / \mathrm{PD}$ & $\mathrm{PR} / \mathrm{R}$ \\
\hline
\end{tabular}

Table I

CONSIDERED RATIOS

the probabilistic peak rate solution. Therefore, we consider 10 ratios that drive our comparison as shown in table (I).

\section{A. Sceanrio I}

In this scenario we consider that $50 \%$ of users are closer to the $802.11 \mathrm{~g}$ AP. For all the considered ratios given in table (I), we show the 95\% confidence interval for scenario I in Figure 2. The intervals in blue represent the ratios of the proposed

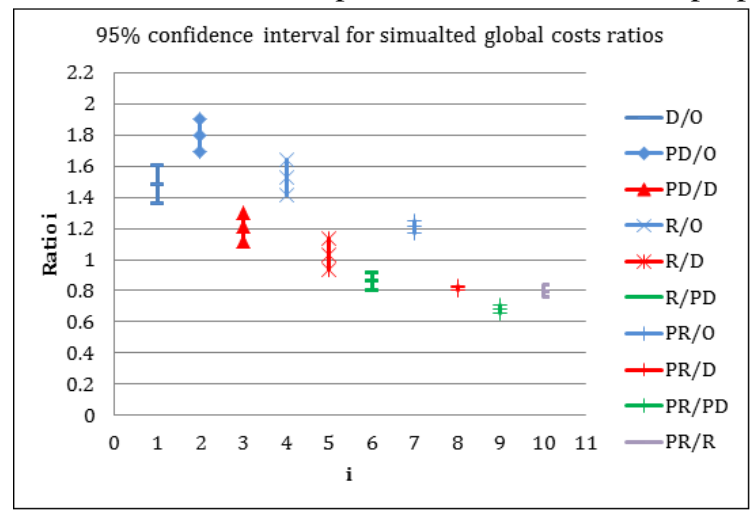

Figure 2. Compared costs for scenario I

algorithms costs with respect to the optimal one (Ratios 1,2, 4 and 7). The red intervals represent the ratios of the heuristic costs with respect to the distance based solution (Ratios 3, 5 and 8). The green ones represent the ratios with respect to the probabilistic distance based (Ratio 6 and 9). Finally, the purple interval represents the cost of probabilistic peak rate cost over the peak rate one (Ratio 10). Note that when the ratio interval is higher than 1 , we can conclude that the solution corresponding to the denominator is more efficient than the one corresponding to the numerator and vice versa. Simulation results confirm that the optimal solution is the most efficient solution that is why all blue intervals are above 1 . Moreover, we see that ratio 7 has an average value of 1.2 so we conclude that the probabilistic peak rate based solution in this scenario is the closest to the optimal one. For the red intervals, ratio 3 interval is above 1 so the distance based solution here is more efficient than the probabilistic distance one since users are in this scenario evenly distributed among the two APs. Ratio 5 has an average value almost close to 1 with a narrow interval. Therefore, we consider that the peak rate based and the distance based solutions present the same efficiency. This result is expected since the 2 APs have the same transmit power, which implies that distance and peak rate are equivalent. From the purple interval (below 1), we affirm that probabilistic peak rate based solution is more efficient than the peak rate based solution. This is due to the fact that the peak rate based solution causes the underutilization of the 
802.11b technology while the probabilistic peak rate based solution avoids the rush to the AP that offers better peak rate by adding uncertainty to the selection so that not all users will be connected to the broader technology.

\section{B. Sceanrio II}

In scenario II, we proceed as in scenario I but here we consider different spatial user distribution so that $75 \%$ of users are closer to the $802.11 \mathrm{~g}$ AP. The obtained results are shown in Figure 3. The blue intervals are all above 1 and confirm that

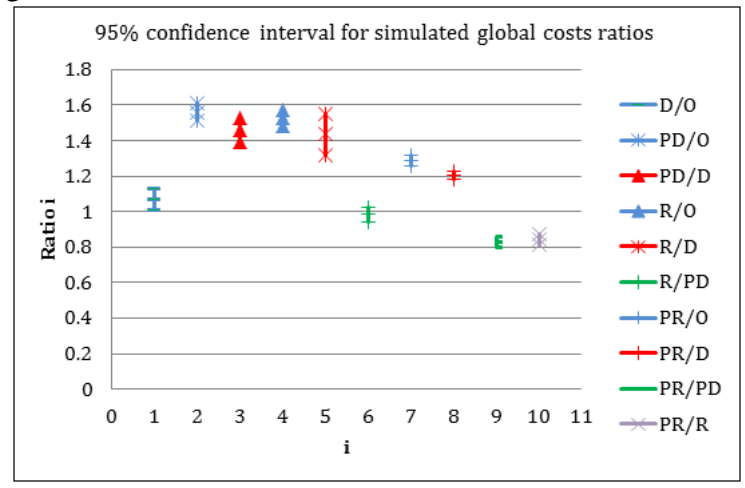

Figure 3. Compared costs for scenario II

the optimal solution is again the most efficient one. Moreover, we see that ratio 1 has an average value close to 1 therefore the distance based solution gives results closest to the optimal one in this scenario. This is predictable since $75 \%$ of users are closer to the $802.11 \mathrm{~g}$ AP, thus they will be well served by this AP given the high capacity offered by $802.11 \mathrm{~g}$ technology. For the green intervals, they are both under the value 1 , therefore in this scenario the peak rate based and the probabilistic peak rate based solutions outperform the probabilistic distance one.

\section{Sceanrio III}

In this scenario, we consider that $75 \%$ of users are closer to the 802.11b AP. We obtain the results shown in Figure 4.

Ratio 1 has an average value of 2.36 therefore the distance

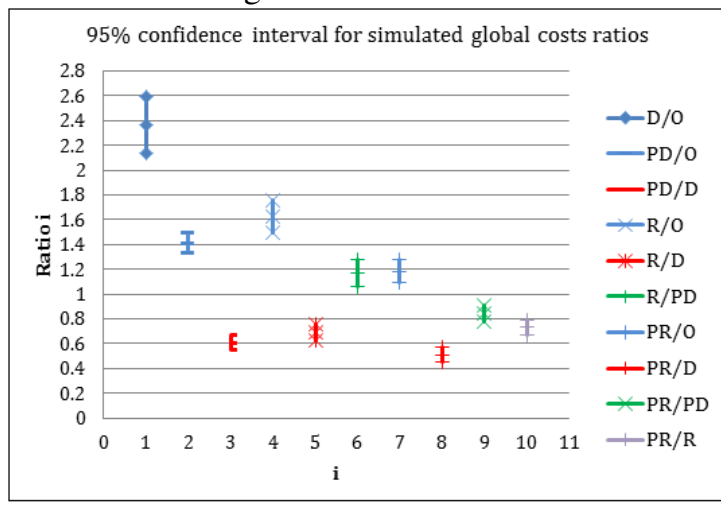

Figure 4. Compared costs for scenario III

based solution in this scenario is not efficient compared to the optimal one. In fact, with $75 \%$ of users connected to $802.11 \mathrm{~b}$ AP, congestion will occur causing degradation of users QoS. Ratio 2 has an average value of 1.4 with a narrow confidence interval, so we can say that the probabilistic distance based solution gives us here good results. Consequently, by combining probability with distance in this scenario we give the possibility to users to connect to the $802.11 \mathrm{~g}$ technology and therefore enhance global performance. For ratio 9, the entire confidence interval is under the value 1 , thus the probabilistic peak rate based solution for this scenario is more efficient than the probabilistic distance one.

\section{CONCLUSION}

In this paper, we described a hybrid system model for RAT selection where the system manages the users RAT association to enhance global performance. Then we studied the case where we have two types of WiFi technology $(802.11 \mathrm{~b}$ and $802.11 \mathrm{~g}$ ) as if we had two different RATs, to find that in the optimal solution of this approach each user is connected to a single RAT. Due to the high computational complexity of the global optimal solution for a large number of users, we proposed four distributed heuristic algorithms for RAT selection. We concluded that each heuristic algorithm presents efficient results depending on the users distribution so it is interesting to implement an entity that chooses the appropriate algorithm. Mainly, the probabilistic peak rate based solution gave us results close to the optimal one. Moreover, a comparison between the proposed algorithms was provided in the three different scenarios. For future work, we suggest to implement these algorithms to the WiMAX - WiFi scenario since WiMAX offers high data rates. This will be an extension of the current work because the model we developed for the global approach treat the fair rate scheduler model for the primary and secondary RAT, thus it is interesting to evaluate these heuristics in the case of the fair time or fair rate - fair time hybrid schedulers since the global optimal solution will boil down to a unique RAT selection.

\section{REFERENCES}

[1] J. Perez-Romero, X. Gelabert, and O. Sallent, Resource Management for Heterogeneous Wireless Access Networks, Heterogeneous Wireless Access Networks Architectures and Protocols, chapter 5, pp. 133163, Springer, 2008.

[2] J. Perez-Romero, O. Sallent, R. Agusti, and M. A. Diaz-Guerra, Radio Resource Management Strategies in UMTS: John Wiley \& Sons, 2005.

[3] A. Tolli, P. Hakalin, and H. Holma, Performance Evaluation of Common Radio Resource Management (CRRM), IEEE International Conference on Communications, USA ,2002 , pp. 3429- 3433.

[4] J. Perez-Romero, O. Sallent, and R. Agusti, Policy-based Initial RAT Selection algorithms in Heterogeneous Networks, MWCN, Morocco, 2005, pp. 1-5.

[5] D. Kumar, E. Altman, J.M Kelif, User-Network Association in an 802.11 WLAN \& 3G UMTS Hybrid Cell: Individual Optimality, USA, 2007.

[6] K. Khawam, M. Ibrahim, J. Cohen, S. Lahoud, S. Tohme, Individual vs. Global Radio Resource Management in a Hybrid Broadband Network, ICC 2011.

[7] I.F. Akyildiz, S. Mohanty, and J. Xie, A ubiquitous mobile communication architecture for next-generation heterogeneous wireless systems, IEEE Radio communications, June 2005.

[8] Wireless LAN Medium Access Control (MAC) and Physical Layer (PHY) specifications, ANSI/IEEE Std 802.11, 2007 Edition.

[9] M. Heusse, F. Rousseau, G. Berger-Sabbatel, and A. Duda, Performance Anomaly of 802.11 b, Proc. IEEE INFOCOM 2003, pp. 836843. 